\title{
A.A.Danilenko, S.V.Shakhtarina \\ Early and long-term pulmonary complications of radiotherapy of Hodgkin's lymphoma
}

Key words: Hodgkin's lymphoma, lung radiation injury, pneumonitis, pulmonary fibrosis.

Ключевые слова: лимфома Ходжкина, лучевые повреждения легких, пневмонит, пневмофиброз.

\section{Патогенез лучевых повреждений легких}

В настоящее время лечение большинства больных лимфомой Ходжкина представляет собой комбинированную химиолучевую терапию. Как и цитостатики, ионизирующая радиация способна оказывать неблагоприятное воздействие на любые органы, в т. ч. легкие.

Так как основной функцией легких является газообмен, то эти органы восприимчивы как к внешним, так и внутренним воздействиям воздуха и крови соответственно. Эволюционные изменения привели к определенному структурному и физиологическому балансу между пассажем воздуха, дыхательной паренхимой и сосудистой системой. Одним из следствий этого является высокая чувствительность респираторной системы к множеству физических, химических и биологических агентов, способных сравнительно легко нарушить тонкий функциональный баланс [1].

Исходом обычного течения раневого процесса является полное восстановление ткани. Однако чрезмерное воздействие патологического агента может запустить каскад преобразований, ведущих к развитию хронического воспалительного процесса, кульминацией которого становится патологическая перестройка ткани [2].

Образующиеся в результате воздействия ионизирующего излучения свободные радикалы лишь в небольшой степени оказывают прямой повреждающий эффект на клетку. Основное повреждение клетка получает вследствие реализации каскада молекулярных событий, запущенных свободными радикалами [3, 4]. Хронический оксидативный стресс, являющийся следствием дисбаланса между количеством молекул реактивного кислорода, азота и антиоксидантной емкостью клетки, приводит в конечном итоге к переокислению жировых молекул, нарушению проницаемости сосудов, отеку, миграции моноцитов, нарушению функции эндотелия, воспалению и формированию фиброза. Воспалительный процесс, со- провождающийся на фоне тканевой гипоксии пролиферацией фибробластов, стимулирует накопление и активацию макрофагов, профиброгенного трансформирующего фактора роста- $\beta$, обусловленного гипоксией проангиогенного фактора- $\beta_{1}$, и фактора роста эндотелия сосудов. Эти молекулярные и клеточные события приводят к гибели клетки, отложению коллагена и образованию фиброза в облученной ткани [5].

Легкие являются комплексным органом, включающим в себя крупные центральные (трахея, бронхи) и мелкие (бронхиолы) воздухоносные пути, сосудистую сеть и альвеолы, в которых происходит газообмен.

Трахея и крупные бронхи выстланы клетками ворсинчатого эпителия и бокаловидными клетками, вырабатывающими слизь. Клетки слизистой оболочки этих структур могут быть повреждены непосредственно в процессе облучения, что выражается в появлении сухого кашля, болезненности горла. Как правило, слизистая оболочка восстанавливается в ближайшие сроки после прекращения лучевого воздействия [6].

Наиболее восприимчив к воздействию облучения альвеолярно-капиллярный комплекс, являющийся функциональной субъединицей легких. Альвеолы выстланы пневмоцитами 1-го типа, представляющими собой плоские эпителиальные клетки с небольшим количеством цитоплазмы и органелл, поэтому толщина альвеолярной выстилки минимальна [7]. Кроме этих клеток, в выстилке присутствуют пневмоциты 2-го типа, вырабатывающие сурфактант. Повреждение альвеол - основное гистопатологическое изменение легочной ткани вследствие облучения. В острой фазе повреждения повышение проницаемости стенки капилляров приводит к отеку межальвеолярной перегородки и экстравазации сывороточных белков в полость альвеолы. Гибель пневмоцитов 1-го типа вызывает повышение пролифера- 
тивной активности пневмоцитов 2-го типа, что поддерживает целостность альвеолярного эпителия. Повреждение пневмоцитов 2-го типа снижает репарационные возможности и сопровождается дополнительным поступлением сурфактанта в просвет альвеолы. Повышение уровня сурфактанта в альвеоле регистрируется через несколько часов после подведения дозы облучения и может продолжаться в течение 2-6 нед. [8].

Обусловленное воздействием ионизирующей радиации повреждение легочной ткани приводит к нарушению многих физиологических аспектов, включая газообмен, перфузию и вентиляцию. В норме альвеолярная вентиляция и капиллярная перфузия находятся в оптимальном динамическом соотношении. Облучение ведет к снижению их функционального уровня, причем перфузия страдает в большей степени. В результате количество функционирующих альвеол сокращается, что выражается в уменьшении жизненной емкости легких (ЖЕЛ) [9].

\section{Раннее лучевое повреждение легких: лучевой пневмонит}

Ранним проявлением лучевого повреждения легких является лучевой пневмонит, который может проявиться как в процессе лучевой терапии, так и в течение 6 мес. после ее окончания.

Основным методом диагностики лучевого пневмонита является рентгенография легких (обычная или компьютерная), позволяющая оценить структуру и плотность ткани. Рентгенологическая семиотика лучевого пневмонита изучена подробно [10]. Чувствительность компьютерной томографии (KT) выше, чем обычной [10, 11].

Частота выявления острого лучевого пневмонита в процессе лечения больных лимфомой Ходжкина, по данным многих авторов, колеблется от 1 до $37 \%$ в зависимости от чувствительности применяемого метода исследования, популяционной выборки, разовой и суммарной очаговых доз облучения (РОД и СОД соответственно), объема облученной легочной ткани, а также других факторов [10]. Более точными представляются результаты исследований, учитывающих показатели нескольких методов оценки состояния легких $[12,13]$.

Исходя из того, что сопоставление ЖЕЛ до операции и объема резецируемой легочной ткани у больных карциномой легких позволяет прогнозировать вероятность ухудшения функции легких после резекции [14], J.Rubenstein et al. (1988) адаптировали этот метод для определения вероятности развития лучевого пневмонита после облучения средостения [15]. Однако N.Choi et al. (1990), исследовав эффективность этого метода, обнаружили наличие существенной переоценки вероятности развития лучевого поражения легких у больных раком легкого, получивших пострезекционную лучевую терапию [16]. Значительное несоответствие ожидаемого и действительного состояний функции легких после лучевой терапии у 210 больных карциномой легкого было установлено W.Currant et al. (1992) [17]. L.Boersma et al. (1993) предложили другое решение оценки зависимости "доза-эффект" при прогнозировании состояния функции легких через 3-4 мес. после облучения средостения у 25 больных лимфомой Ходжкина [18]. Конвертировав объемное распределение (3D) дозы облучения в один интегральный параметр, авторы установили, что корреляция между стандартными легочными тестами (определение диффузионной способности легких, объема форсированного выдоха) и средним изменением плотности легочной ткани на КТ менее существенна, чем между стандартными тестами и перфузией и вентиляцией легких ( $Q$ и $V$ [19]. Эти же авторы показали, что на 18 -й месяц после лучевой терапии функция легких у больных, перенесших лучевой пневмонит, восстановилась до ее уровня у больных лимфомой Ходжкина, не имевших лучевого пневмонита [20]. Это подтверждает тот факт, что лучевой пневмонит, развивающийся в процессе лучевой терапии или в течение 3-4 мес. после ее завершения, не всегда реализуется в постлучевой пневмофиброз. J.Theuws et al. (1998), изучив изменение функции легких у 41 больного лимфомой Ходжкина через 3-4 мес. после облучения области средостения с подведением различных СОД, установили, что 1 Гр поглощенной легкими дозы облучения коррелирует с ухудшением функции легких на $1 \%$ [21].

\section{Факторы риска развития лучевого пневмонита}

Повышение риска возникновения клинически значимого лучевого пневмонита может быть связано с наличием сопутствующих заболеваний легких, снижением ко времени лучевой терапии функциональной способности легких, тяжелым общим состоянием больного, женским полом, наличием истории табакокурения [22]. Противоречивость результатов соответствующих исследований не позволяет считать эти зависимости безусловными [6]. Более достоверна зависимость риска развития острого лучевого пневмонита от подведенной к участку легкого РОД и СОД облучения относительного объема подвергнутой облучению части легкого [23-26]. Исследование E.S.Koh et al., которые за период с 2003 по 2005 г. наблюдали 64 больных лимфомой Ходжкина, показало, что риск развития клинически значимого лучевого пневмонита возникал при подведении к средостению СОД облучения > 14 Гр при условии включения в поле облучения $>36 \%$ легочной ткани [27].

\section{Роль цитостатиков в развитии лучевого пневмонита}

Известно, что цитостатики, входящие в многокомпонентные схемы терапии лимфомой Ходжкина, обладают спектром токсичности, в т. ч. негативным воздействием на легкие [28]. Учитывая, что современные программы лечения больных лимфомой Ходжкина представляют собой комбинацию последовательного проведения химио- и лучевой терапии, 
вполне вероятно усиление отрицательного воздействия облучения на легочную ткань вследствие предшествовавшей химиотерапии.

Изучение потенцирующего влияния цитостатиков на токсичность лучевой терапии относительно легочной ткани сопряжено с определенными методологическими трудностями. Учитывая, что в клинических центрах лучевые установки отличаются мощностью дозы, отсутствуют стандартные методы формирования поля облучения области средостения, существуют различия в подводимых СОД облучения, то сопоставление результатов, полученных в разных клиниках, проблематично. Такая же проблема может быть и в каждом отдельном клиническом центре вследствие постоянного совершенствования тактики лечения больных лимфомой Ходжкина. Например, в исследовании Г.Д.Байсоголова с соавт. (1985) лучевой пневмонит в группе больных (180 человек), получивших только лучевую терапию, встречался чаще, чем в группе комбинированной химиолучевой терапии (119 пациентов), в которой химиотерапия проводилась по схеме СОРР (циклофосфан, винкристин, натулан, преднизолон). Подведенная к средостению СОД облучения была одинаковой в обеих группах. Авторы объясняли существенное различие в частоте развития лучевого пневмонита тем, что в результате применения химиотерапии объем опухолевой массы сокращался, вследствие чего уменьшалась и площадь поля облучения средостения, в проекции которого находился меньший объем легочной ткани [29].

Влияние химиотерапии на частоту развития лучевого пневмонита удалось оценить A.Hirsch et al. (1996). Для суждения о состоянии легочной ткани исследователи оценили ЖЕЛ и диффузионную способность легких (выделение окиси углерода) у 60 больных лимфомой Ходжкина I-IIIA стадии. Только химиотерапию (схема ABVD: доксорубицин, блеомицин, винкристин, дакарбазин) получили 30 человек, остальные - химиолучевое лечение. В процессе проведения химиотерапии по схеме ABVD с применением стандартных доз цитостатиков (6 циклов) уменьшение дозы или исключение дальнейших введений блеомицина потребовалось по клиническим показаниям 14 пациентам. Последующее мантиевидное облучение проводилось в режиме подведения РОД 1,8 Гр до достижения СОД 36 Гр. Исходный поперечный размер поля облучения средостения соответствовал размеру поперечника тени средостения до начала химиотерапии. При подведении СОД 18 Гр размер поперечника поля облучения сокращался в соответствии с изменением границ опухоли вследствие химиотерапии. У 37 \% пациентов после окончания химиотерапии было зарегистрировано уменьшение ЖЕЛ и диффузионной способности легких. По завершении последовавшей лучевой терапии у 8 \% из них было отмечено дальнейшее ухудшение этих показателей [30].

Противоположный результат получен A.Macann et al. (2008). Проведенное ими исследование было направлено на изучение эффективности и токсич- ности химиотерапии по схеме BAGCOPP (блеомицин, доксорубицин, циклофосфан, винкристин, натулан, гемцитабин, преднизолон) у больных лимфомой Ходжкина II-IV стадии. Набор пациентов в группу исследования был прекращен досрочно в связи с развитием серьезных легочных осложнений в процессе или сразу по окончании химиотерапии (у 8 из 27 отобранных), у 1 из них - со смертельным исходом. Авторы отнесли столь сильный токсический эффект со стороны легочной ткани на счет взаимного потенцирующего влияния блеомицина и гемцитабина. Консолидирующая лучевая терапия с подведением СОД 36 Гр была проведена 15 пациентам, в т. ч. 4 - с предшествовавшим цитостатическим пневмонитом. Интервал между окончанием химиотерапии и началом лучевой терапии составлял 4 нед. Развитие лучевого пневмонита не было зарегистрировано ни у одного из этих 4 больных, вследствие чего авторы предположили, что лучевая терапия на область средостения может быть вполне безопасной при условии соблюдения 4-недельного интервала между окончанием химио- и началом лучевой терапии лимфомы Ходжкина [31]. Похожие результаты получены A.M.Fox et al. (2012). В группе из 75 первичных больных лимфомой Ходжкина, получивших комбинированное химиолучевое лечение, лучевой пневмонит развился в $10 \%$ случаев. Возникновение лучевого пневмонита не было связано с наличием предшествующих блеомициновых пневмонитов. В группе из 17 пациентов, у которых лучевая терапия рецидива лимфомы Ходжкина комбинировалась с высокодозовой терапией, поддержанной трансплантацией стволовых клеток, лучевой пневмонит зарегистрирован в 35 \% случаев, при этом тяжесть претрансплантационных лучевых пневмонитов была существенно выше, чем посттрансплантационных (лучевой пневмонит III степени - у $57 \%$ и $0 \%$ больных соответственно; $p=0,015)$ [26]. Авторы связывают значительное усиление тяжести претрансплантационных пневмонитов с применением кармустина.

\section{Позднее лучевое повреждение легких: пневмофиброз}

Отдаленный эффект воздействия облучения на легочную ткань - пневмофиброз - может оказывать, значительное влияние на качество жизни пациента (в отличие от раннего эффекта) [10]. Первые признаки пневмофиброза можно обнаружить в большинстве случаев не ранее чем через 6 мес. после завершения лучевой терапии средостения. Клиническая значимость пневмофиброза, как правило, обратно пропорциональна периоду между окончанием лучевой терапии и 1-й рентгенологической регистрацией пневмофиброза [32]. Рентгенологическая и клиническая характеристики впервые зарегистрированного пневмофиброза далеко не всегда окончательные, т. к. процесс фиброзирования развернут во времени. Завершение формирования фиброза происходит, как правило, в течение 2 лет после окончания луче- 
вой терапии [33]. Вследствие лучевого пневмонита сокращается ЖЕЛ, страдает диффузионная способность легких, что сопровождается прогрессирующей одышкой. R.Abratt et al. (2002) показали, что смертность больных раком легкого, получавших одинаковую терапию, выше у тех пациентов, у которых диффузионная способность легких в результате лечения ухудшилась [34]. Хотя лучевой пневмонит чаще всего предшествует лучевому пневмофиброзу, непосредственная связь между этими процессами необязательна [35].

Данные о частоте постлучевого пневмофиброза, приведенные в литературе, имеют широкий диапазон значений. В работе Т.А.Коврыжккиной и соавт. (1997) частота пневмофиброза, оцененного рентгенологически у 44 больных лимфомой Ходжкина в диапазоне от 5 мес. до 6 лет после подведения к средостению СОД 40 Гр, составила $93 \%$, при этом частота фиброза III степени - 11,4 \% [36]. Похожие результаты были получены F.Villani et al. (2009). Авторы обследовали 126 больных лимфомой Ходжкина спустя 5 лет после окончания химиолучевой терапии, включавшей облучение средостения. Больные получили химиотерапию по одной из 3 схем: ABVD (доксорубицин, блеомицин, винбластин, дакарбазин), ABVD-MOPP (мехлоретамин, винкристин, натулан, преднизолон) или VЕВЕРР (винкристин, эпидоксорубицин, блеомицин, циклофосфан, этопозид, преднизолон). СОД облучения средостения составила 36-40 Гр, в зависимости от исходных размеров медиастинальных лимфатических узлов. Постлучевой пневмофиброз III степени, сопровождавшийся существенным ухудшением функции легких, был обнаружен у 18 (14 \%) пациентов. Авторы не указали частоту менее интенсивного пневмофиброза [37]. Экстраполируя результаты, полученные T.A.Коврыжкиной [36], на когорту F.Villani et al., можно предполагать, что пневмофиброз сформировался практически у всех пациентов этого исследования. Вероятнее всего, именно пневмофиброз явился основной причиной наличия у многих больных (число не уточнялось) хронической обструктивной болезни легких [37].

J.Theuws et al. (1998), изучив перфузию, вентиляцию легких, изменение плотности легочной ткани на КТ 41 больного лимфомой Ходжкина в промежутке времени до 4 лет после химиолучевой терапии, установили, что лучевые повреждения легких, зарегистрированные через 3-4 мес. после терапии, регрессировали частично или полностью через 18 мес. у $50 \%$ пациентов. Через 48 мес. после терапии улучшения не регистрировались [21].

M.B.Lund et al. (1999) изучили результаты терапии 116 больных лимфомой Ходжкина через 5-13 лет после химио(ABVD)-лучевого лечения, при котором подведенная к средостению СОД составила 36-40 Гр. Частота пневмофиброза достигла $68 \%$; в $12 \%$ случаев заболевание сопровождалось ухудшением функции легких [38].

В исследовании R.M.Enrichi et al. (1999) частота пневмофиброза оказалась относительно невысокой у 12 (16,4 \%) пациентов. Рентгенологическое исследование легких было проведено в периоде наблюдения (медиана - 114 мес.) после лучевого и химиолучевого лечения 73 больных лимфомой Ходжкина (37 из них получили только лучевую терапию, а у $36-$ лучевой терапии предшествовал 1 цикл химиотерапии ABVD). В обоих случаях СОД области средостения составила 36-40 Гр. Фиброз был зарегистрирован у 2 пациентов, получивших только лучевое лечение, и у 10 больных, получавших комбинированную химиолучевую терапию. В связи с относительно небольшим числом пациентов различие не оказалось достоверным. Все случаи пневмофиброза не имели клинического значения [39]. В работе E.Brusamolino et al. (2006) частота пневмофиброза после комбинированной химио- (4 цикла ABVD) лучевой (СОД - 30-36 Гр) терапии 112 пациентов с лимфомой Ходжкина составила $8 \%$ (9 человек) с медианой времени до выявления 76 (50-123) недель. Клинически значимый пневмофиброз развился у 3 больных. Относительно малая частота пневмофиброза в этом исследовании может быть связана с отсутствием пациентов с массивной медиастинальной опухолевой массой и, соответственно, меньшим объемом облучения области средостения. Это дало основание авторам полагать, что частота пневмофиброза связана не с СОД, а с размером поля облучения [40]. Однако представленные в этой работе данные не позволяют сопоставить эти зависимости.

Наименьшая частота постлучевого пневмофиброза спустя 10 лет после терапии лимфомы Ходжкина у $6(6,4 \%)$ из 94 пациентов была получена в исследовании J.M.Andrieu et al. (1998). Все больные получили 3 цикла химиотерапии по схеме ABVD и лучевую терапию, включавшую облучение средостения с подведением СОД 40 Гр [41].

Учитывая идентичность схем химиотерапии и СОД облучения средостения в указанных выше исследованиях, можно предполагать, что столь большой диапазон частоты постлучевого пневмофиброза связан с различиями рентгенологических критериев, использованных в разных центрах, соотношением больных с массивной и умеренной медиастинальной опухолевой массой, количеством предшествовавших лучевой терапии циклов химиотерапии по схеме ABVD, принципами формирования поля облучения средостения, с продолжительностью наблюдения за пациентами после лечения лимфомы Ходжкина.

Необходимо отметить, что данные о частоте развития постлучевого пневмофиброза были получены в когортах больных, получивших СОД на область средостения 36-40 Гр. Влияние меньшей (20-30 Гр) СОД на частоту развития пневмофиброза оценено в работе А.А.Даниленко и соавт. (2010). Частота пневмофиброза в парамедиастинальных отделах легких среди 180 больных лимфомой Ходжкина, получивших только лучевое лечение с подведением к области средостения 40 Гр, составила 95 \%; среди 119 больных, получивших химиолучевое лечение с такой же дозой облучения - 77,3 \%; в когорте из 192 больных, 
получивших химиолучевую терапию с подведением СОД 20-30 Гр, пневмофиброз развился у 38 \% пациентов [42].

\section{Терапевтические подходы к предупреждению лучевого поражения легких}

Так как лучевые повреждения легких способны не только существенно влиять на качество жизни, но и могут привести к смерти пациента, в т. ч. получавшего лечение по поводу лимфомы Ходжкина, вполне оправданным является поиск молекулярных мишеней и, соответственно, создание лекарственных препаратов, способных уменьшать неблагоприятное воздействие ионизирующей радиации на легкие.

Одним из очевидных терапевтических направлений является воздействие на 1-е звено лучевого патогенеза - образование свободных радикалов. Десятилетия исследовательских работ привели к созданию и внедрению в клинику препарата амифостин, показавшего свою эффективность в качестве антиоксиданта [43]. Учитывая, что результаты, оцененные в процессе клинических исследований, существенно уступали предварительным, полученным на кроликах, можно предполагать, что конкурентными "потребителями" амифостина являются печень и почки, в результате чего накопление амифостина в легких оказалось менее ожидаемого [44].

Исследуется также возможность усиления энзимной системы защиты организма от оксидативного повреждения. Эта система включает в себя выработку и активацию каталазы, глютатионовой пероксидазы и супероксидных дисмутаз [45]. Серьезное внимание исследователей привлекают супероксидные дисмутазы. Наиболее перспективным способом повышения уровня супероксидных дисмутаз энзимов представлялась модификация соответствующих генов, однако в связи с неудачными попытками проведения в предыдущем десятилетии генной терапии энтузиазм исследователей существенно уменьшился. Тем не менее продолжаются работы по поиску возможности влиять на уровень супероксидных дисмутаз другими способами [46].

Еще одним направлением является воздействие на воспалительный процесс. Преклинические исследования таких противовоспалительных препаратов, как ингибитор циклооксигеназы-2 целекоксиб и индометацин, не привели к положительным результатам. В противоположность им статины, противовоспалительный эффект которых уже показан при некоторых заболеваниях, заслуживают определенного внимания. Активность одного из статинов ловастатина - была протестирована на мышах, легкие которых были облучены. В группе мышей, получавших ловастатин, частота лучевого пневмонита оказалась ниже, а выживаемость животных - выше [47].

Попытки уменьшить влияние фактора некроза опухоли- $\alpha$ отражены в крайне малом количестве работ, а полученные результаты - далеки от обнадеживающих [48].
Роль интерлейкина-1 (IL-1) в воспалительном процессе, в т. ч. в патогенезе пневмонита, изучена во многих работах. В настоящее время проводится клиническое исследование способности антагониста IL-1 кинерета уменьшать частоту и выраженность лучевого пневмонита и пневмофиброза.

Существовавшее долгое время представление о том, что применение кортикостероидов для лечения лучевого пневмонита препятствует формированию пневмофиброза, не нашло подтверждения, в то время как серьезные осложнения кортикостероидной терапии лучевого пневмонита продолжают встречаться [49].

\section{Заключение}

Таким образом, наиболее клинически значимым осложнением лучевой терапии области средостения является фиброз легких, способный ухудшать качество и уменьшать продолжительность жизни пациента. Следовательно, факторам, влияющим на риск развития пневмофиброза, необходимо уделять пристальное внимание при планировании лучевой терапии области средостения.

\section{Литература}

1. Williams J.P., Johnston C.J., Finkelstein J.N. Treatment for radiation-induced pulmonary late effects: Spoiled for choice or looking in the wrong direction? Curr. Drug Targets 2010; 11 (11): 1386-1394.

2. Notter R.H., Finkelstein J.N., Holm B.A. Lung injury: mechanisms, pathophysiology, and therapy. New York: Taylor \& Fransis, 2005.

3. Bentzen S.M. Preventing or reducing late side effects of radiation therapy: Radiobiology meets molecular pathology. Nature Rev. Cancer. 2006; 6: 702-713.

4. Mikkelsen R.B., Wardman P. Biological chemistry of reactive oxygen and nitrogen and radiation-induced signal transduction mechanisms. Oncogene 2003; 22: 5734-5754.

5. Fleckenstein K., Zgonjanin L., Chen L. et al. Temporal onset of hypoxia and oxidative stress after pulmonary irradiation. Int. J. Radiat. Oncol. Biol. Phys. 2007; 68: 196-204.

6. Ghafoori P., Marks L.B., Vujaskovic Z. et al. Radiationinduced lung injury: assessment, management, and prevention. Oncology 2008; 22 (1): 37-47.

7. Young B., Lowe J.S., Stevens A., Heath J.W. Wheather's functional histology: a text and colour atlas. 5rd ed. London: Churchill Livingstone, 2006.

8. Rubin P., Shapiro D.L., Finklestein J.N. et al. The early release of surfactant following lung irradiation of alveolar type II cells. Int. J. Radiat. Oncol. Biol. Phys. 1980; 6: 75-77.

9. Allavena C., Conroy T., Aletti P. et al. Late cardiopulmonary toxicity after treatment for Hodgkin's disease. Br. J. Cancer 1992; 65: 908-912.

10. Torrisi J.M., Schwartz L.H., Gollub M.J. CT findings of chemotherapy-induced toxicity: what radiologists need to know about the clinical and radiologic manifestations of chemotherapy toxicity. Radiology 2011; 258 (1): 41-56.

11. Трахтенберг А.Х., Чиссов В.И. Клиническая онкопульмонология. М.: ГЭОТАР Медицина, 2000.

12. Marks L.B., Fan M., Clough R. et al. Radiation-induced pulmonary injury: Symptomatic versus subclinical endpoints. Int. J. Radiat. Biol. 2000; 76: 469-475. 
13. Goethals I., Dierckx R., Meerleer G. et al. The role of nuclear medicine in the prediction and detection of radiation-associated normal pulmonary and cardiac damage. J. Nucl. Med. 2003; 44 (9): 1531-1539.

14. Bria W.F., Kanarek D.J., Kazemi H. Prediction of postoperative pulmonary function following thoracic operations: value of ventilation-perfusion scanning. J. Thorac. Cardiovasc. Surg. 1983; 86: 186-192.

15. Rubenstein J., Richter M., Moldofsky P. et al. Prospective prediction of post-radiation therapy lung function using quantitative lung scans and pulmonary function testing. Int. J. Radiat. Oncol. Biol. Phys.1988; 15: 83-87.

16. Choi N., Kanarek D., Grillo H. Effect of post-operative radiotherapy on changes in pulmonary function in patients with stage II and IIIa lung carcinoma. Int. J. Radiat. Oncol. Biol. Phys.1990; 18: 95-99.

17. Curran W., Moldofsky P., Solin L. Observations on the predictive value of perfusion lung scans on post-irradiation pulmonary function among 210 patients with bronchogenic carcinoma. Int. J. Radiat. Oncol. Biol. Phys.1992; 24: $31-36$.

18. Boersma L., Damen.E., de Boer R. et al. A new method to determine dose-effect relations for local lung function changes using correlated SPECT and CT data. Radiother. Oncol.1993; 29: 110-116.

19. Boersma L., Damen E., de Boer R.W. et al. Estimation of overall pulmonary function after irradiation using doseeffect relations for local functional injury. Radiother. Oncol. 1995; 36: 15-23.

20. Boersma L., Damen E., de Boer R.W. et al. Recovery of overall and local function loss 18 monthsafter irradiation for malignant lymphoma. J. Clin. Oncol.1996; 14: 1431-1441.

21. Theuws J., Kwa S., Wagenaar A. et al. Prediction of overall pulmonary function loss in relation to 3-D dose distribution for patients with breast cancer and malignant lymphoma. Radiother. Oncol. 1998; 49: 233-243.

22. Robnett T.J., Machtay M., Vines E.P. et al. Factors predicting severe radiation pneumonitis in patients receiving definitive chemoradiation for lung cancer. Int. J. Radiat. Oncol. Biol. Phys.2000; 48: 89-94.

23. Kim T.H., Cho K.H., Pyo H.R. et al. Dose-volumetric parameters for predicting severe radiation pneumonitis after three-dimentional conformal radiation therapy for lung cancer. Radiology 2005; 235: 208-215.

24. Kong F.M., Haymann J.A., Griffith K.A. et al. Final toxicity results of a radiation-dose escalation study in patients with non-small-cell lung cancer (NSCLC): Predictors for radiation pneumonitis and fibrosis. Int. J. Radiat. Oncol. Biol. Phys. 2006; 65: 1075-1086.

25. Hua C., Hoth K.A., Wu S. et al. Incidence and correlates of radiation pneumonitis in pediatric patients with partial lung irradiation. Int. J. Radiat. Oncol. Biol. Phys. 2012; 78 (1): 43-149.

26. Fox A.M., Dosoretz A.P., Mauch P.M. et al. Predictive factors for radiation pneumonitis in Hodgkin is lymphoma patients receiving combined modality therapy. Int. J. Radiat. Oncol. Biol. Phys. 2012; 83 (1): 277-283.

27. Koh E.S., Sun A., Tran T.H. et al. Clinical dose-volume histogram analysis in predicting radiation pneumonitis in Hodgkin's lymphoma. Int. J. Radiat. Oncol. Biol. Phys. 2006; 66 (1): 223-228.

28. Meadors M., Floyd J., Perry M.C. Pulmonary toxicity of chemotherapy. Semin. Oncol. 2006; 33: 98-105.

29. Байсоголов Г.Д., Афанасова Н.В., Шахтарина С.В. и др. Изменения в легких у больных лимфогранулематозом
I-II клинической стадии после комбинированного лечения. Мед. радиол. 1985; 3: 18-22.

30. Hirsch A., Van der Els N., Straus D.J. et al. Effect of ABVD chemotherapy with and without mantle or mediastinal irradiation on pulmonary function and symptoms in early-stage Hodgkin`s disease. J. Clin. Oncol. 1996; 14: 1297-1305.

31. Macann A., Bredenfeld H., Muller R.P. et al. Radiotherapy does not influence the severe pulmonary toxicity observed with the administration of gemcitabine and bleomycin in patients with advanced-stage Hodgkin's lymphoma treated with the BAGCOPP regimen: a report by the German Hodgkin's Lymphoma Study Group. Int. J. Radiat. Oncol. Biol. Phys. 2008; 70 (1): 161-165.

32. Бардычев М.С., Цыб А.Ф. Местные лучевые повреждения. М.: Медицина, 1985.

33. Park K.J., Chung J.Y., Chun M.S. et al. Radiation-induced lung disease and the impact of radiation methods on imaging features. Radiographics 2000; 20 (1): 83-98.

34. Abratt R., Morgan G. Lung toxicity following chest irradiation in patients with lung cancer. Lung Cancer 2002; 35: 103-109.

35. Rubin P., Finkelstein J., Shapiro D. Molecular biology mechanisms in the radiation induction of pulmonary injury syndromes: interrelationship between the alveolar macrophage and the septal fibroblasts. Int. J. Radiat. Oncol. Biol. Phys. 1992; 24: 93-101.

36. Коврыжкина Т.А., Ильин Н.В., Акимов А.А. и др. Использование линейно-квадратичной модели для оценки поздних лучевых повреждений легких у больных лимфогранулематозом. Мед. радиол. 1997; 3: 59-63.

37. Villani F., Busia A., Villani M. et al. Cardiopulmonary response to exercise in patients with different degrees of lung toxicity after radio-chemotherapy for Hodgkin's disease. Anticancer Res. 2009; 29 (2): 777-784.

38. Lund M.B., Kongerud J., Boe J. et al. Late complications after treatment of Hodgkin`s disease. Tidsskr. Norske Laegeforen. 1999; 119 (7): 933-937.

39. Enrichi R.M., Anselmo A.P., Donato V. et al. Relapse and late complications in early-stage Hodgkin's disease patients with mediastinal involvement treated with radiotherapy alone or plus one cycle of ABVD. Haematologica 1999; 84 (10): 17-23.

40. Brusamolino E., Baio A., Orlandi E. et al. Long-term events in adult patients with clinical stage IA-IIA nonbulky Hodgkin's lymphoma treated with four cycles of doxorubicin, bleomycin, vinblastine, and dacarbazine and adjuvant radiotherapy: a single-institution 15 -year follow-up. Clin. Cancer Res. 2006; 12 (21): 6487-6493.

41. Andrieu J.M., Jais J.P., Colonna P. et al. Ten-year results of a strategy combining three cycles of ABVD and high-dose extended irradiation for treating Hodgkin's disease at advanced stages. Ann. Oncol. 1998; 9 (2): 195-203.

42. Даниленко А.А., Шахтарина С.В., Афанасова Н.В., Павлов В.B. Изменения в легких у больных лимфомой Ходжкина после химиотерапии по схемам СОРР, ABVD, BEACOPР и облучения средостения в суммарной очаговой дозе 20-30 Гр. Клин. онкогематол. 2010; 3 (4): 354-358.

43. Sasse A.D., Clark L.G., Sasse E.C., Clark O.A. Amifostine reduces side effects and improves complete response rate during radiotherapy: results of a meta-analysis. Int. J. Radiat. Oncol. Biol. Phys. 2006; 64: 784-791.

44. Levi M., Knol J.A., Ensminger W.D. et al. Regional pharmacokinetics of amifostine in anesthetized dogs: role of the liver, gastrointestinal tract, lungs, and kidneys. Drug Metabol. Disposit. 2002; 30: 1425-1430. 
45. Greenberg J.S., Epperly M.W. Antioxidant gene therapeutic approaches to normal tissue protection and tumor radiosensitization. In Vitro 2007; 21: 141-146.

46. Gauter-Fleckenstein B., Fleckenstein K., Owzar K. et al. Comparison of two Mn porphyrin-based mimics of superoxide dismutase in pulmonary radioprotection. Free Radic. Biol. Med. 2008; 44: 982-989.

47. Williams J.P., Hernady E., Johnston C.J. et al. Effect of administration of lovastatin on the development of late pulmonary effects following whole lung irradiation in a murine model. Radiat. Res. 2004; 161: 560-567.

48. Xie C.H., Zhang M.S., Zhou Y.F. et al. Chinese medicine Angelica sinensis supresses radiation-induced expression of TNF- $\alpha$ and TGF- $\beta$ in mice. Oncol. Rep. 2006; 15: $1429-1436$.
49. Kozaka Y., Mitsumori M., Araki N. et al. Avascular necrosis of bilateral femoral head as a result of long-term steroid administration for radiation pneumonitis after tangential irradiation of the breast. Int. J. Clin. Oncol. 2006; 11: 482-486.

\section{Информация об авторах}

Даниленко Анатолий Александрович - к. М. Н., старший научный сотрудник отделения лучевой и лекарственной терапии гемобластозов ФГБУ "Медицинский радиологический научный центр" Минздрава России; тел.: 8-909-250-02-22; e-mail: danilenko@mrrc.obninsk.ru Шахтарина Светлана Васильевна - д. М. н., ведущий научный сотрудник отделения лучевой и лекарственной терапии гемобластозов ФГБУ "Медицинский радиологический научный центр" Минздрава России; тел.: (48439) 9-31-01; e-mail: danilenko@mrrc.obninsk.ru

Поступила 25.03.13 () Даниленко А.А., Шахтарина С.В., 2013 удк 616-006.443-085.849.1-06 